\title{
Zooarqueología de la transición prehispánica y posthispánica del sitio arqueológico Casa de Piedra de Ortega (Río Negro, Argentina)
}

\section{( Ailín A. Guillermo*}

Fecha de defensa: 6 de Julio de 2017

Director: Dr. Fernando J. Fernández

Codirector: Dr. José A. Cordero

Jurados: Dres. Mariana De Nigris y Pablo M.

Fernández

En este trabajo se dan a conocer los resultados del análisis de los conjuntos arqueofaunísticos provenientes del sitio arqueológico Casa de Piedra de Ortega (CPO), provincia de Río Negro (Patagonia, Argentina, 40 $43^{\prime} 49^{\prime \prime}$ 'S y $70^{\circ} 42^{\prime} 21$ " O; $675 \mathrm{~m}$ s.n.m.). Se estudian los conjuntos pertenecientes a períodos prehispánicos y posthispánicos. El primero comprende el Período III de CPO $\left(1490 \pm 70\right.$ años ${ }^{14} \mathrm{C}$ AP, LP1320) y el segundo el Período IV (280 \pm 50 años ${ }^{14} \mathrm{C}$ AP, LP191) y el Período V. Este último, presenta taxones exóticos domésticos, por lo tanto, CPO nos permite caracterizar el impacto de la fauna exótica (Equus caballus, Bos taurus y Ovis aries) en la subsistencia de los grupos humanos que habitaron la zona de estudio luego del contacto hispanoindígena. En este sentido, los objetivos específicos de este trabajo incluyen 1) determinar la composición taxonómica de los conjuntos estudiados, 2) evaluar la integridad y resolución de los mismos, 3) explorar los patrones de obtención, procesamiento, consumo y descarte que los grupos humanos implementaron sobre las especies exóticas y 4) reconocer diferencias en el aprovechamiento de, respectivamente, la fauna exótica y la autóctona, principalmente Lama guanicoe.

\section{Área de estudio}

CPO es una pequeña cueva orientada al oeste que se encuentra en la cuenca superior del río Limay en Río Negro en el noroeste de Patagonia. El área de estudio comprende las zonas del interior e interfluvios del arroyo Pichileufú, en el paraje Corralito, hasta su desembocadura en el río Limay en el Departamento de Pilcaniyeu. El Pichileufú es un arroyo permanente de caudal variable que recorre unos $160 \mathrm{~km}$ de extensión con dirección suroeste-noreste desde Corralito hasta Paso Flores. El clima es árido. La precipitación anual media es de $250 \mathrm{~mm}$. La temperatura anual media es de 10 a $14{ }^{\circ} \mathrm{C}$. Domina el viento del sector oeste y sudoeste. Fitogeográficamente, pertenece a una área de mosaico entre la estepa arbustiva-graminosa del Distrito Occidental, la estepa arbustiva del Distrito Central

* Centro de Investigaciones en Antropología Filosófica y Cultural. Av. Federico Lacroze 2100 (CP C1426CPS), Ciudad Autónoma de Buenos Aires, Argentina. E-mail: ailin.guillermo@gmail.com y la estepa arbustiva del Ecotono Rionegrino (León, Bran, Collantes, Paruelo y Soriano, 1998).

\section{Metodología}

Desde una perspectiva tafonómica, se registraron rastros de actividad de carnívoros (Binford, 1981) y de roedores (Lyman, 1994; Politis y Madrid, 1988), los resultados de la acción de aves rapaces (Fernández, Montalvo, Fernández-Jalvo, Andrews y López, 2017), las marcas de raíces (Montalvo, 2002), los indicadores de pisoteo (Shipman, 1981), la diagénesis (Lyman, 1994) y el grado de meteorización del conjunto (Behrensmeyer, 1978).

La identificación y cuantificación anatómica y taxonómica se realizó mediante la utilización de colecciones óseas de referencia y atlas osteológicos (Pacheco Torres, Alfredo y Guerra Porras, 1979). La frecuencia taxonómica y de partes esqueletales se calcularon a partir de: Número de Especímenes Identificados por Taxón, Número Mínimo de Individuos, Número Mínimo de Elementos y Número Mínimo de Unidades Anatómicas (Lyman, 1994; Mengoni Goñalons, 1988, 1999, 2006).

Se identificaron huellas de corte, raspado, machacado, percusión y negativos de impacto (Blumenschine, Marean y Capaldo, 1996). También se infirió la finalidad de las huellas de corte (Binford, 1981).

Para el análisis de fracturas (Mengoni Goñalons, 1999; Shipman y Rose, 1983) se consideraron atributos relacionados con el tipo de fractura primaria (espiral, transversal y longitudinal), el tipo de borde que presenta la fractura (regular, astillado o con marcado perimetral) y el estado en que se encontraban los huesos antes de la fractura (fresco/no fresco).

El análisis de la termoalteración se realizó según el atributo basado en el color (Shipman, Foster y Schoeninger, 1984): no quemado (blanco), quemado incipiente (marrón claro), quemado avanzado (marrón oscuro), carbonizado (negro) y calcinado (blanco azulado).

La frecuencia relativa de partes esqueletales se correlacionó con el índice de utilidad para el guanaco calculado por Borrero (1990) y modificado por Lyman (1992) y para la oveja (Binford, 1978). También se correlacionaron los valores entre la frecuencia relativa de partes y la densidad mineral ósea (Elkin, 1995). 
Para estimar la diversidad taxonómica de cada conjunto se utilizó el índice de Shannon $(\mathrm{H})$ sobre la base del Número de Especímenes Identificados por Taxón (Grayson, 1984; Lyman, 2008).

\section{Resultados}

La baja incidencia de agentes naturales (meteorización, erosión, acción de carnívoros, por ejemplo) indicó una buena conservación y rápido enterramiento de los conjuntos arqueofaunísticos estudiados beneficiados por las características intrínsecas del sitio. No se descarta la presencia de actividades de pisoteo evidenciadas por las fracturas en especímenes en estado no fresco y por la capa de guano consolidado en el Período V.

Una parte considerable de los restos arqueofaunísticos ingresaron impulsados por actividades humanas, incluyendo la explotación de fauna autóctona y exótica para alimentación y usos de las pieles.

Lama guanicoe, Rhea pennata y Conepatus chinga se explotaron continuamente a lo largo de los tres períodos de CPO, aunque Lama guanicoe fue el principal recurso en todas las ocupaciones. En el Período IV, Lycalopex griseus, Lycalopex culpaeus y Chaetophractus villosus se incluyeron en la alimentación de los grupos humanos. En el Período V, se registró la incorporación de Equus caballus, Bos taurus y Ovis aries.

En las ocupaciones posthispánicas de CPO se observó un aumento en la intensidad de explotación de Lama guanicoe como así también una intensificación por diversificación taxonómica, evidenciada en la explotación de nuevos taxones, no utilizados en el último período prehispánico. La especie exótica más explotada fue Ovis aries. La comparación con el aprovechamiento de Lama guanicoe indicó similitudes y diferencias.

Bos taurus y Equus caballus fueron incluidos en la alimentación pero de un modo minoritario en relación con Ovis aries. Por lo tanto, se plantea que no hubo un reemplazo de la explotación de la fauna autóctona con la llegada de la fauna exótica doméstica. Equus caballus, Bos taurus y Ovis aries se incorporaron a la dieta de los habitantes de CPO de modo complementario. La comparación de los resultados de CPO con otros sitios arqueológicos con contextos prehispánicos y posthispánicos ubicados en el área de estudio (Valle Encantado 1 y La Marcelina 1), indicó prácticas de subsistencia similares.

\section{Referencias citadas}

"Behrensmeyer, A. (1978). Taphonomic and ecologic information from bone weathering. Paleobiology, 4, 150-162.
" Binford, L. (1978). Nunamiut Ethnoarchaeology. Nueva York: Academic Press.

" Binford, L. (1981). Bones: Ancient Men and Modern Myths. Nueva York: Academic Press.

» Blumenschine, R. J., Marean, C. W. y Capaldo, S. D. (1996). Blind test of inter-analyst correspondence and accuracy in the identification of cut marks, percussion marks, and carnivore tooth marks on bone surfaces. Journal of Archaeological Science, 23, 493-507.

"Elkin, D. (1995). Volume density of South American camelid skeletal parts. International Journal of Osteoarchaeology, 5(1), 29-37.

"Fernández, F. J., Montalvo, C. I., Fernández-Jalvo, Y., Andrews, P. y López, J. M. (2017). A re-evaluation of the taphonomic methodology for the study of small mammal fossil assemblages of South America. Quaternary Science Review, 155, 37-49.

" Grayson, D. (1984). Quantitative Zooarcheology. Topics in the Analysis of Archaeological Faunas. Nueva York: Academic Press.

" León, R., Bran, D., Collantes, M., Paruelo, J. y Soriano, A. (1998). Grandes unidades de vegetación de la Patagonia extra andina. Ecología Austral, 8, 125-144.

" Lyman, R. (1992). Prehistoric seal and sea-lion butchering on the Southern Northwest Coast. American Antiquity, 57(2), 246-261.

" Lyman, R. (1994). Vertebrate Taphonomy. Cambridge: Cambridge University Press.

" Lyman, R. (2008). Quantitative Paleozoology. Cambridge: Cambridge University Press.

" Mengoni Goñalons, G. L. (1988). Análisis de materiales faunísticos de sitios arqueológicos. Xama, 1, 71-120.

" Mengoni Goñalons, G. L. (1999). Cazadores de Guanacos de la Estepa Patagónica. Buenos Aires: Sociedad Argentina de Antropología.

»Mengoni Goñalons, G. L. (2006). Zooarqueología en la práctica: algunos temas metodológicos. Xama, 19, 83-113.

" Montalvo, C. (2002). Root traces in fossil bones from the huayquerian (Late Miocene) faunal assemblage of Telén, La Pampa, Argentina. Acta Geológica Hispánica, 37(1), 37-42.

》Pacheco Torres, V., Alfredo, A. y Guerra Porras, E. (1979). Guía Osteológica para Camélidos Sudamericanos. Lima: Universidad Nacional de Mayor de San Marcos.

"Politis, G. y Madrid, P. (1988). Un hueso duro de roer: análisis preliminar de la tafonomía del sitio Laguna 
Tres Reyes I (Pdo. de A. González Chaves, Pcia. de Buenos Aires). En N. Ratto y A. Haber (Eds.), De Procesos, Contextos y otros Huesos (pp. 29-44). Buenos Aires: Instituto de Ciencias Antropológicas.

»Shipman, P., Foster, G. y Schoeninger, M. (1984). Burnt bones and teeth: An experimental study of color, morphology, crystal structure and shrinkage. Journal of Archaeological Science, 11, 307-325.

"Shipman, P. y Rose, J. (1983). Early hominid hunting, butchering and carcass-processing behaviors approaches to the fossil record. Journal of Antropological Archaeology, 2, 57-98. 